\title{
Using characteristics of the residuals as an assessment of the effectiveness of processing the results of measurements
}

\author{
Vladimir Marchuk ${ }^{1, *}$, Alexander Minaev ${ }^{1}$, and Igor Shrafel ${ }^{1}$ \\ ${ }^{1}$ Don State Technical University, 344000 Rostov-on-Don, Russia
}

\begin{abstract}
The article considers an analytical assessment of the use of characteristics of residuals for evaluating the efficiency of processing measurement results. The proved is provided that the method of reproduction of estimates is highly effective in processing the measurement results.
\end{abstract}

\section{Introduction}

At present, in practical implementation of the processing of measurement results, in many cases, analysis of residuals is used as a criterion for extracting a useful signal [1-4]. This is due to the fact that any model, however accurate it may be, assumes the presence of residuals - deviations of the theoretical values of a series from empirical data. In the absence of a useful component model, the standard deviation criterion is usually used. The application of this criterion is widely used in practice. In this regard, we will prove analytically using the example that using the method of reproduction of estimates of the useful component [5-12] allows the dispersion of residuals to be approximated to the value of the dispersion of the additive noise component. It should be noted that in terms of efficiency, this criterion is inferior to the criterion for minimizing the root-mean-square error, using the useful component model, since it requires a priori information about the dispersion of the additive noise component and there is no pronounced minimum error.

\section{Research}

In this paper, we will consider the case of calculating the variance of the estimate of the constant signal by the method of multiplying the estimates with a random division of the implementation of the original process into two parts [5-6]. Define arbitrary positive integers $n, l, m$ such that

$$
m \leq n-2 l+1
$$

\footnotetext{
* Corresponding author: marchuk@sssu.ru
} 
and consider a set of $n$ pairwise uncorrelated random variables $x_{1}, \ldots, x_{n}$ with equal mathematical expectations and equal variances: $M x_{k}=a, D x_{k}=D, 1 \leq k \leq n$. The value $a$ will be considered unknown, and the random vector $\left(x_{1}, \ldots, x_{n}\right)$ - the sum of the constant signal $a$ and noise at the moments of time $t_{1}<\ldots<t_{n}$. As a result we get the implementation of a random process $\left(x_{k}\right)_{k=1}^{n}$. Estimate the value $a$ using the method of reproduction estimates. We randomly divide $m$ times the set $\{1, \ldots, n\}$ into two parts $U_{j}$, $V_{j}$, so that for all the following conditions are met:

1) $\left|U_{j}\right| \geq l,\left|V_{j}\right| \geq l$, (hereinafter $|A|$ is the number of elements of the set $A$ );

2) $\max U_{j}<\min V_{j}$;

3) $U_{j} \neq U_{i}, i=1, \ldots, j-1$ (with $j=1$ );

4) the probability of choosing a pair $U_{j}, V_{j}$ is considered equal for all pairs sets that satisfy conditions 1-3.

From inequality (1) it follows that the described $m$-parts partition of the set $\{1, \ldots, n\}$ is always possible. Estimation $a_{k j}$ of the signal $a$ in the $k$-th section $(1 \leq k \leq n)$ at the multiplication of estimates $j \quad(1 \leq j \leq m)$, is the average value of the estimates of this signal found by the least squares method (LSM) in the part $\left(x_{i}\right)_{i \in T_{j}}$ of the original implementation, where through $T_{j}$ the one of the sets $U_{j}, V_{j}$ the number belongs to is denoted. In our case, the LSM are used - estimates of zero degree polynomials:

$$
\bar{a}_{k j}=\frac{1}{\left|T_{j}\right|} \sum_{i \in T_{j}} x_{i}, \text { then } \bar{a}_{k}=\frac{1}{m} \sum_{j=1}^{m} a_{k j}
$$

Obviously, $\bar{a}_{k j}$, and therefore $\bar{a}_{k}$ are unbiased estimates of $a$. The result of a $m$-times division of the set $\{1, \ldots, n\}$ into parts $U_{j}, V_{j}$, that satisfy conditions $1-4$, can be represented as a random chosen subset $A$ with $m$ elements of the set $\{l, \ldots, n-l\}$, each element $p$ of which symbolizes a certain pair of sets $U_{j}=\{1, \ldots, p\}, V_{j}=\{p+1, \ldots, n\}$. Because of condition 4 , the random choice $A$ has a uniform distribution law. Consequently, the probability $P$ of its results is the inverse of the number of ways in which such a choice can be made $P=\frac{1}{C_{n-2 l+1}^{m}}$. The average variance $D_{c p}=\frac{1}{n} \sum_{k=1}^{n} D_{a_{k}}$ is used as the measure of the quality of the assessment multiplication method. It is necessary to calculate $D_{c p}$ and prove that with constant $n, l$ and increasing $m$ quality of processing improves, i.e. $D_{c p}$ decreases. Note that inequality $n \geq 2 l$ follows from (1). 


\section{Solution}

The trivial case $n=2 l$ was considered in [6]; therefore, we consider only the main case $n \geq 2 l+1$. The sum $\sum_{j=p}^{q}$ at $p>q$ is considered equal to zero. Fix arbitrary $1 \leq k \leq n$. Now

$$
\begin{aligned}
a_{k}= & \frac{1}{m} \sum_{j=1}^{m} a_{k j}=\frac{1}{m} \sum_{i=1}^{n} x_{i}\left(\sum_{\substack{j=l \\
j \in A}}^{\min (i, k)-1} \frac{1}{n-j}+\sum_{\substack{j=\max (i, k) \\
j \in A}}^{n-l} \frac{1}{j}\right)= \\
& =\frac{1}{m} \sum_{i=1}^{m} x_{i}\left(\sum_{j=l}^{\min (i, k, n-l)-1} \frac{\tau_{j}}{n-j}+\sum_{j=\max (i, k, l)}^{n-l} \frac{\tau_{j}}{j}\right)
\end{aligned}
$$

where $\tau_{j}=|A \bigcap\{j\}|$. The first sum in brackets can be converted to $\sum_{j=n+1-\min (i, k, n-l)}^{n-l} \frac{\tau_{n-j}}{j}$.

Consequently, $\quad a_{k}=\frac{1}{m} \sum_{i=1}^{n} x_{i} y_{k i}$, where $\quad y_{k i}=\sum_{j=\max (n-i, n-k, l)+1}^{n-l} \frac{\tau_{n-j}}{j}+\sum_{j=\max (i, k, l)}^{n-l} \frac{\tau_{j}}{j}$. Then

$$
D_{a_{k}}=M\left[\left(\frac{1}{m} \sum_{i=1}^{m} x_{i} y_{k i}\right)^{2}\right]-\left(M_{a_{k}}\right)^{2}=M\left(\frac{1}{m^{2}} \sum_{i, p=1}^{n} x_{i} x_{p} y_{k i} y_{k p}\right)-a^{2}
$$

Given the independence of random variables $x_{i} x_{p}$ and $y_{k i} y_{k p}$ and pairwise uncorrelated random variables $x_{1}, \ldots, x_{n}$, we obtain

$$
\begin{gathered}
D_{a_{k}}=\frac{1}{m^{2}} \sum_{i, p=1}^{n} M\left(x_{i} x_{p}\right) M\left(y_{k i} y_{k p}\right)-a^{2}= \\
=\frac{1}{m^{2}}\left(\sum_{i, p=1}^{n} M\left(x_{i}^{2}\right) M\left(y_{k i}^{2}\right)+\sum_{\substack{i, p=1 \\
i \neq p}}^{n} M_{x_{i}} M_{x_{p}} M\left(y_{k i} y_{k p}\right)\right)-a^{2}= \\
=\frac{1}{m^{2}}\left(\sum_{i=1}^{n}\left(D+a^{2}\right) M\left(y_{k i}^{2}\right)+a^{2} \sum_{\substack{i, p=1 \\
i \neq p}}^{n} M\left(y_{k i} y_{k p}\right)\right)-a^{2}= \\
=\frac{1}{m^{2}}\left(D \sum_{i=1}^{n} M\left(y_{k i}^{2}\right)+a^{2} \sum_{i, p=1}^{n} M\left(y_{k i} y_{k p}\right)\right)-a^{2}=
\end{gathered}
$$




$$
\begin{gathered}
=\frac{D}{m^{2}} \sum_{i=1}^{n} M\left(y_{k i}^{2}\right)+\frac{a^{2}}{m^{2}} M\left(\sum_{i, p=1}^{n} M\left(y_{k i} y_{k p}\right)\right)-a^{2}= \\
=\frac{D}{m^{2}} \sum_{i=1}^{n} M\left(y_{k i}^{2}\right)+\frac{a^{2}}{m^{2}} M\left(\left(\sum_{i, p=1}^{n} y_{k i}\right)^{2}\right)-a^{2}=\frac{D}{m^{2}} \sum_{i=1}^{n} M\left(y_{k i}^{2}\right),
\end{gathered}
$$

because $\frac{1}{m} \sum_{i=1}^{n} y_{k i}$ is the sum of random coefficients of a linear function $a_{k}=a_{k}\left(x_{1}, \ldots, x_{n}\right)$, which is always equal to 1 . Find the expected value of random variables $\tau_{i}, \tau_{j}(l \leq i, j \leq n-l)$ with $i=j \quad \tau_{i} \tau_{j}=\tau_{i}^{2}=\tau_{i}$

$$
M\left(\tau_{i}^{2}\right)=\frac{C_{n-2 l}^{m-1}}{C_{n-2 l+1}^{m}}=\frac{(n-2 l) ! m !(n-2 l+1-m) !}{(m-1) !(n-2 l-m+1) !(n-2 l+1) !}=\frac{m}{n-2 l+1}
$$

If $i \neq j$, then

$$
M\left(\tau_{i} \tau_{j}\right)=\frac{C_{n-2 l-1}^{m-2}}{C_{n-2 l+1}^{m}}=\frac{(n-2 l-1) ! m !(n-2 l+1-m) !}{(m-2) !(n-2 l-m+1) !(n-2 l+1) !}=\frac{m(m-1)}{(n-2 l+1)(n-2 l)}
$$

This expression is also true when $m=1$, because then $\tau_{i}, \tau_{j}=0$ with probability 1 . For all $\tau_{i}, \tau_{j}=0$ we have

$$
\begin{aligned}
& M\left(y_{k i}^{2}\right)=M\left[\left(\sum_{j=\max (n-i, n-k, l)+1}^{n-l} \frac{\tau_{n-j}}{j}\right)^{2}+\left(\sum_{j=\max (i, k, l)}^{n-l} \frac{\tau_{j}}{j}\right)^{2}+2 \sum_{j=\max (n-i, n-k, l)+1}^{n-l} \frac{\tau_{n-j}}{j} \sum_{j=\max (i, k, l)}^{n-l} \frac{\tau_{n-j}}{j}\right]= \\
& =M\left[\sum_{j, p=\max (n-i, n-k, l)+1}^{n-l} \frac{\tau_{n-j} \tau_{n-p}}{j p}+\sum_{j, p=\max (i, k, l)}^{n-l} \frac{\tau_{j} \tau_{p}}{j p}+2 \sum_{j=\max (n-i, n-k, l)+1}^{n-l} \sum_{p=\max (i, k, l)}^{n-l} \frac{\tau_{n-j} \tau_{p}}{j p}\right]= \\
& =\sum_{j, p=\max (n-i, n-k, l)+1}^{n-l} \frac{M\left(\tau_{n-j} \tau_{n-p}\right)}{j p}+\sum_{j, p=\max (i, k, l)}^{n-l} \frac{M\left(\tau_{j} \tau_{p}\right)}{j p}+2 \sum_{j=\max (n-i, n-k, l)+1}^{n-l} \sum_{p=\max (i, k, l)}^{n-l} \frac{M\left(\tau_{n-j} \tau_{p}\right)}{j p}= \\
& =\frac{m}{n-2 l+1}\left(\sum_{j=\max (n-i, n-k, l)+1}^{n-l} \frac{1}{j^{2}}+\sum_{j=\max (i, k, l)}^{n-l} \frac{1}{j^{2}}\right)+\frac{m(m-1)}{(n-2 l+1)(n-2 l)} \times \\
& \times\left(\sum_{\substack{j, p=\max \\
j \neq p}}^{n-l} \frac{1}{j p-i, n-k, l)+1}+\sum_{\substack{j, p=\max (i, k, l) \\
j \neq p}}^{n-l} \frac{1}{j p}+2 \sum_{j=\max (n-i, n-k, l)+1}^{n-l} \sum_{p=\max (i, k, l)}^{n-l} \frac{1}{j p}\right)=
\end{aligned}
$$




$$
\begin{aligned}
= & \frac{m(n-2 l-m+1)}{(n-2 l+1)(n-2 l)}\left(\sum_{j=\max (n-i, n-k, l)+1}^{n-l} \frac{1}{j^{2}}+\sum_{j=\max (i, k, l)}^{n-l} \frac{1}{j^{2}}\right)+\frac{m(m-1)}{(n-2 l+1)(n-2 l)} \times \\
& \times\left(\sum_{j, p=\max (n-i, n-k, l)+1}^{n-l} \frac{1}{j p}+\sum_{j, p=\max (i, k, l)}^{n-l} \frac{1}{j p}+2 \sum_{j=\max (n-i, n-k, l)+1}^{n-l} \sum_{p=\max (i, k, l)}^{n-l} \frac{1}{j p}\right) .
\end{aligned}
$$

Sum up by $i, k$ from 1 to $n$ each of the five sums in brackets.

$$
\begin{gathered}
\sum_{i, k=1}^{n} \sum_{j=\max (n-i, n-k, l)+1}^{n-l} \frac{1}{j^{2}}=\sum_{j=l+1}^{n-l} \frac{1}{j^{2}} \sum_{i, k=n-j+1}^{n} 1=\sum_{j=l+1}^{n-l} 1=n-2 l ; \\
\sum_{i, k=1}^{n} \sum_{j=\max (i, k, l)+1}^{n-l} \frac{1}{j^{2}}=\sum_{j=l}^{n-l} \frac{1}{j^{2}} \sum_{i, k=1}^{j} 1=\sum_{j=l}^{n-l} 1=n-2 l+1 ; \\
\sum_{i, k=1}^{n} \sum_{j, p=\max (n-i, n-k, l)+1}^{n-l} \frac{1}{j p}=\sum_{j, p=l+1}^{n-l} \frac{1}{j p} \sum_{i, k=\max (n-j, n-p)+1}^{n} 1= \\
=\sum_{j, p=l+1}^{n-l} \frac{(\min (j, p))^{2}}{j p}=\sum_{j, p=l+1}^{n-l} \frac{\min (j, p)}{\max (j, p)}=\sum_{j=l+1}^{n-l} \frac{\min (j, j)}{\max (j, j)}+\sum_{j, p=l+1}^{n-l} \frac{p}{j}+\sum_{j, p=l+1}^{n-l} \frac{j}{j<p} \\
=\sum_{j=l+1}^{n-l} 1+2 \sum_{j, p=l+1}^{n-l} \frac{p}{j}=n-2 l+2 \sum_{j=l+2}^{n-l} \frac{1}{j} \sum_{p=l+1}^{j-1} p=n-2 l+\sum_{j=l+2}^{n-l} \frac{(j+l)(j-l-1)}{j}= \\
=n-2 l+\sum_{j=l+2}^{n-l}(j-1)-l(l+1) \sum_{j=l+2}^{n-l} \frac{1}{j}=n-2 l+\frac{n(n-2 l-1)}{2}-l(l+1) \sigma_{l+2}=
\end{gathered}
$$

(hereafter the designation $\sigma_{j}=\sum_{i=j}^{n-l} \frac{1}{i}$ is used; note that when $j>n-l \quad \sigma_{j}=0$ )

$$
\begin{gathered}
=\frac{n^{2}+n-2 l \cdot n-4 l}{2}-l(l+1) \sigma_{l+2} ; \\
\sum_{i, k=1}^{n} \sum_{j, p=\max (i, k, l)}^{n-l} \frac{1}{j p}=\sum_{j, p=l}^{n-l} \frac{1}{j p} \sum_{i, k=1}^{\min (j, p)} 1=\sum_{j, p=l}^{n-l} \frac{(\min (j, p))^{2}}{j p}= \\
=\sum_{j, p=l+1}^{n-l} \frac{(\min (j, p))^{2}}{j p}+\frac{(\min (l, l))^{2}}{l^{2}}+\sum_{p=l+1}^{n-l} \frac{l^{2}}{l p}+\sum_{j=l+1}^{n-l} \frac{l^{2}}{j l}=
\end{gathered}
$$




$$
\begin{gathered}
=\frac{n^{2}+n-2 l \cdot n-4 l}{2}-l(l+1) \sigma_{l+2}+1+2 l \sigma_{l+1} ; \\
\sum_{i, k=1}^{n} \sum_{j=\max (n-i, n-k, l)+1}^{n-k} \frac{1}{j} \sum_{h=\max (i, k, l)}^{n-l} \frac{1}{p}=\sum_{j=l+1}^{n-l} \frac{1}{j} \sum_{p=l}^{n-l} \frac{1}{p} \sum_{i, k=n+1-j}^{p} 1= \\
=\sum_{j=l+1}^{n-l} \frac{1}{j} \sum_{p=\max (l, n+1-j)}^{n-l} \frac{p-n+j}{p}=\sum_{j=l+1}^{n-l} \frac{1}{j} \sum_{p=n+1-j}^{n-l} 1+\sum_{j=l+1}^{n-l} \sum_{p=n+1-j}^{n-l} \frac{1}{p}-n \sigma=
\end{gathered}
$$

(Hereafter $\left.\sigma=\sum_{j=l+1}^{n-l} \frac{1}{j} \sum_{p=n+1-j}^{n-l} \frac{1}{p}\right)$

$$
=\sum_{j=l+1}^{n-l} \frac{j-l}{j}+\sum_{p=l+1}^{n-l} \frac{1}{p} \sum_{j=n+1-p}^{n-l} 1-n \sigma=2 \sum_{j=l+1}^{n-l} 1-2 l \sigma_{l+1}-n \sigma=2 n-4 l-2 l \sigma_{l+1}-n \sigma .
$$

Thereby,

$$
\begin{gathered}
D_{c p}=\frac{1}{n} \sum_{k=1}^{n} D_{a_{k}}=\frac{D}{n m^{2}} \sum_{i, k=1}^{n} M\left(y_{k i}^{2}\right)=\frac{D}{n m(n-2 l+1)(n-2 l)} \cdot[(n-2 l-m+1) \\
\cdot(n-2 l+n-2 l+1)+(m-1)\left(2\left(\frac{n^{2}+n-2 l \cdot n-4 l}{2}-l(l+1) \sigma_{l+2}\right)+1+2 l \sigma_{l+1}+\right. \\
\left.\left.+2\left(2 n-4 l-2 l \sigma_{l+1}-n \sigma\right)\right)\right]=\frac{D}{n m(n-2 l+1)(n-2 l)}[(n-2 l)(2 n-4 l+1)+(m-1) . \\
\left.\cdot\left(-2 n+4 l-1+n^{2}+n-2 l \cdot n-4 l+4 n-8 l-2 l(l+1) \sigma_{l+2}-2 l \sigma_{l+1}-2 n \sigma+1\right)\right]= \\
\left.\left.-2 l\left(\sigma_{l+2}+\frac{1}{l+1}\right)-2 n \sigma\right)\right]=\frac{D}{n m(n-2 l+1)(n-2 l)}[(n-2 l)(2 n-4 l+1)+(m-1) \times \\
\left.\times\left(n^{2}-2 l \cdot n+3 n-8 l-\frac{2 l}{l+1}-2 l(l+2) \sigma_{l+2}-2 n \sigma\right)\right]
\end{gathered}
$$

Let us prove that with an increase in the number of reproduction estimates $m$, the mean variance decreases. The resulting ratio is

$$
D_{c p}=\frac{\alpha}{m}(\beta+(m-1) \gamma)
$$




\section{EEESTS-2019}

where $\alpha, \beta$ and $\gamma$ do not depend on $m$. Now it is required to prove inequality

$$
\frac{\alpha}{m}(\beta+(m-1) \gamma)>\frac{\alpha}{m+1}(\beta+m \gamma) \Leftrightarrow(m+1)(\beta+(m-1) \gamma)>m(\beta+m \gamma) \Leftrightarrow \beta>\gamma,
$$

i.e. inequality

$$
\begin{gathered}
(n-2 l)(2 n-4 l+1)>n^{2}-2 l \cdot n+3 n-8 l-\frac{2 l}{l+1}-2 l(l+2) \sigma_{l+2}-2 n \sigma \Leftrightarrow \\
\Leftrightarrow 2 n^{2}-4 l \cdot n+n-4 l \cdot n+8 l^{2}-2 l-n^{2}+2 l \cdot n-3 n+8 l+\frac{2 l}{l+1}+2 l(l+2) \sigma_{l+2}+2 n \sigma> \\
>0 \Leftrightarrow n^{2}-6 l \cdot n+8 l^{2}-2 n+6 l+\frac{2 l}{l+1}+2 l(l+2) \sigma_{l+2}+2 n \sigma>0 .
\end{gathered}
$$

For each $l \geq 1$, we prove the increase of the sequence $\left(\phi_{n}\right)_{n=2 l+1}^{\infty}$, where $\phi_{n}$ is the left side of inequality (2), reduced by $2 n \sigma$. With any $n \geq 2 l+1$ we get

$$
\begin{gathered}
\phi_{n+1}=\phi_{n}=(n+1)^{2}-6 l(n+1)+8 l^{2}-2(n+1)+6 l+\frac{2 l}{l+1}+2 l(l+2) \sum_{j=l+2}^{n+1-l} \frac{1}{j}- \\
-n^{2}+6 l \cdot n-8 l^{2}+2 n-6 l-\frac{2 l}{l+1}-2 l(l+2) \sum_{j=l+2}^{n+l} \frac{1}{j}=2 n+1-6 l-2+\frac{2 l(l+2)}{n+1-l}= \\
=\frac{(2 n-6 l-1)(n+1-l)+2 l^{2}+4 l}{n+1-l}=\frac{2 n^{2}-8 l \cdot n+8 l^{2}+n-l-1}{n+1-l}= \\
=\frac{2(n-2 l)^{2}+n-l-1}{n+1-l}>\frac{0+2 l+1-l-1}{n+1-l}>0 .
\end{gathered}
$$

Thus, the required thesis is proved. Now we find

$$
\begin{aligned}
\phi_{2 l+1}= & (2 l+1)^{2}-6 l(2 l+1)+8 l^{2}-2(2 l+1)+6 l+\frac{2 l}{l+1}+2 l(l+2) \sum_{j=l+2}^{l+1} \frac{1}{j}= \\
= & 4 l^{2}+4 l+1-12 l^{2}-6 l+8 l^{2}-4 l-2+6 l+\frac{2 l}{l+1}=\frac{l-1}{l+1} \geq 0
\end{aligned}
$$

From all this it follows that $\phi_{n} \geq 0$ for all $n \geq 2 l+1$. Note also that with the same values $n \quad \sigma=\sum_{j=l+1}^{n-l} \frac{1}{j} \sum_{p=n+1-j}^{n-l} \frac{1}{p}>\frac{1}{(n-l)^{2}}>0$. Therefore $\phi_{n}+2 n \sigma>0$, i.e. inequality (9) holds for all $n \geq 2 l+1$. Thus, with increasing $m$ value $D_{c p}$ decreases. 
In practice, in many cases, the residual analysis serves as a criterion for the quality of models, which allows to determine whether a series of residuals is Gaussian noise or if there are any interdependencies in its structure.

\section{Conclusion}

1. It is analytically proved that when using the method of reproduction of estimates for extracting a signal under conditions of a priori uncertainty, the value $D_{c p}$ decreases with $m$ increasing.

2. The obtained ratios make it possible to use comparative analysis of residual characteristics (correlation function, differential distribution density, etc.) as quality criteria for processing measurement results.

\section{References}

1. S.M. Perevertkin, A.V. Kantor, N.F. Borodin, T.S. Shcherbakova, Onboard telemetry equipment of spacecraft (Mashinostroenie, Moscow, 1977)

2. T. Anderson, Statistical analysis of time series (Mir, Moscow, 1976)

3. J. Bendat, A. Pirsol, Applied analysis of random data (Mir, Moscow, 1989)

4. M. Kendall, J. A. Stuart, Statistical findings and communication (Science, Moscow, 1973)

5. V.I. Marchuk, Primary processing of measurement results with a limited amount of a priori information: Monograph (Publishing house TRGU, Taganrog, 2003)

6. V.I. Marchuk, I.S. Shrayfel, Methods for isolating the useful component with a priori uncertainty and a limited amount of measurement results. (Publishing House SSSU Shakhty, 2007)

7. V.I. Marchuk, Measuring equipment, 12, 3 (2003)

8. V.I. Marchuk, K.E. Rumyantsev, Aerospace Instrument Engineering, 4, 48 (2004)

9. V.I. Marchuk, G.R. Saakyan, Review of Applied and Industrial Mathematics, 9, 1, 126 (2002)

10. V.I. Marchuk, O.V. Saakyan, G.R. Saakyan, News of universities. North Caucasus region. Technical science, 3, 67 (1999)

11. M. A. Budin, IEEE Transactions on systems, MAN, and cybernetics, 4, 371 (1973)

12. V.I. Marchuk, Serbian journal of electrical engineering, 15, 3, 365 (2018) 